\title{
Computer Assisted Drug Design of Larotrectinib for treatment of Cancer: A Combined Density Functional and Molecular Docking Study
}

\author{
Shamim Molla \\ Department of Pharmacy, Bangabandhu Sheikh Mujibur Rahman Science and Technology University
} (BSMRSTU)

\author{
Abul Bashar Ripon Khalipha* \\ Department of Pharmacy, Bangabandhu Sheikh Mujibur Rahman Science and Technology University \\ (BSMRSTU)
}

Evergreen Scientific Research Center (ESRC), Bagnabandhu Sheikh Mujibur Rahman Science \& Technology University

Md. Solayman Hossain, Sajal Biswas

Department of Pharmacy, Bangabandhu Sheikh Mujibur Rahman Science and Technology University

(BSMRSTU)

\begin{abstract}
This article talks about hypothesis behind the most critical strategies and later effective uses of halogencoordinated Larotrectinib, ligand-based techniques utilize just ligand data for foreseeing action contingent upon its similitude/uniqueness to recently known dynamic ligands. We audit generally utilized ligand-based techniques, for example, ligand-based pharmacophores, atomic descriptors, and quantitative structure-action connections. What's more, vital instruments, for example, target/ligand information bases, homology displaying, ligand ADMET and so on. And fundamental for fruitful usage of different PC supported medication revelation/structure strategies in best simple of Larotrectinib disclosure are talked about. At long last, computational strategies for poisonous quality expectation and improvement for positive physiologic properties are talked about with fruitful lead for Larotrectinib from writing. The helpful capability of Larotrectinib has been examined widely and the dynamic mixes of Larotrctinib are appeared to be engaged with adjusting different physiological reactions. In addition this article will audit the structure of arrangement of halogen-coordinated tinosporides before outline on how the atoms apply their capacities by means of cooperations with different flag transducer and activator proteins of interpretation which were planned by homology demonstrating. Procedures for CADD change contingent upon the degree of basic and other data accessible in regards to the objective (chemical/receptor) and the ligands. The procedure by which another Larotrectinib item is conveyed to showcase arrange is alluded to by various names most normally as the improvement chain and comprises of various unmistakable stages.
\end{abstract}

Keywords: CADD; ADMET; Molecular Modeling; Larotrectinib.

DOI: $10.7176 / \mathrm{JNSR} / 9-9-08$

Publication date:May $31^{\text {st }} 2019$

\section{Introduction}

TRK fusions, involving the genes NTRK1, NTRK2, and NTRK3, occur in a broad range of solid tumors and are oncogenic.Targeted inhibition of TRK can be an effective antitumor approach. Larotrectinib is the first selective pan-TRK inhibitor in clinical development and has demonstrated an overall response rate of $76 \%$ by investigator assessment [1] The incidence of TRK fusions in TC has been reported to be as high as $27 \%$ in pediatric patients and $12 \%$ in adult patients, especially in tumors with papillary histology[2] In SGCs, TRK fusions define mammary analog secretory cancers (MASC) [3] Here we provide the first report summarizing the activity and safety of TRKdirected therapy with larotrectinib in the treatment of advanced, recurrent TRK-fusion TCs and SGCs including nonpapillary and non-MASC histologies. Larotrectinib is an orally available, tropomyosin receptor kinase (Trk) inhibitor, with potential antineoplastic activity. Upon administration, larotrectinib binds to Trk, thereby preventing neurotrophin-Trk interaction and Trk activation, which results in both the induction of cellular apoptosis and the inhibition of cell growth in tumors that over express Trk. Trk, a receptor tyrosine kinase activated by neurotrophins, is mutated in a variety of cancer cell types and plays an important role in tumor cell growth and survival.[4] To determine the anti-cancer activity of Larotrectinib and its halogenated derivatives, computational tools have been used to investigate the best ligand and optimized receptors protein as the best choice for the future scientists. The position of $-\mathrm{OH}$ group in the chemical structure of Larotrectinib was carbon-10 that was changed to carbon- 8 position as main compound and also the $-\mathrm{OH}$ group in carbon-8 was modified with $-\mathrm{F},-\mathrm{Cl},-\mathrm{Br},-\mathrm{I}$ and $-\mathrm{CF} 3$ for the identification of hit -to -lead. 
2. Drug profile: Larotrectinib is a highly potent small-molecule inhibitor of TRKA, TRKB, and TRKC (nM IC50 in cellular assays)[5][11.]Highly selective, with little or no interaction with other kinase and non-kinase targets18. Liquid formulation allows dosing of children as young as 1 month of age and delivers equivalent PK to capsules. Larotrectinib is highly active against TRK fusion cancers with Durable responses in both children and adults.[1]

\subsection{Chemical and Physical properties:}

\begin{tabular}{|l|l|}
\hline Property Name & Property Value \\
\hline Molecular Weight & $428.444 \mathrm{~g} / \mathrm{mol}$ \\
\hline Hydrogen Bond Donor Count & 2 \\
\hline Hydrogen Bond Acceptor Count & 7 \\
\hline Rotatable Bond Count & 3 \\
\hline Complexity & 659 \\
\hline Topological Polar Surface Area & $86 \mathrm{~A}^{\wedge} 2$ \\
\hline Monoisotopic Mass & $428.177 \mathrm{~g} / \mathrm{mol}$ \\
\hline Exact Mass & $428.177 \mathrm{~g} / \mathrm{mol}$ \\
\hline XLogP3-AA & 1.7 \\
\hline Compound Is Canonicalized & true \\
\hline Formal Charge & 0 \\
\hline Heavy Atom Count & 31 \\
\hline Defined Atom Stereocenter Count & 2 \\
\hline Undefined Bond Stereocenter Count & 0 \\
\hline Covalently-Bonded Unit Count & 1 \\
\hline
\end{tabular}

\subsection{Pharmacology and Biochemistry:}

Larotrectinib is an orally available, tropomyosin receptor kinase (Trk) inhibitor, with potential antineoplastic activity. Upon administration, larotrectinib binds to Trk, thereby preventing neurotrophin Trk interaction and Trk activation, which results in both the induction of cellular apoptosis and the inhibition of cell growth in tumors that overexpress Trk. Trk, a receptor tyrosine kinase activated by neurotrophins, is mutated in a variety of cancer cell types and plays an important role in tumor cell growth [https://ncit.nci.nih.gov/ncitbrowser/ConceptReport.jsp?dictionary=NCI_Thesaurus\&ns=NCI_Thesaurus\&code= C115977]

\subsection{Drug Indication:}

a) Treatment of salivary gland cancer

b) Treatment of glioma

c) Treatment of papillary thyroid cancer

\subsection{TRK fusions are rare but recurrent oncogenic drivers:}

Beyond embryogenesis, tropomyosin receptor kinase (TRK) protein expression is primarily limited to the nervous system [11] neurotrophin receptors encoded by 3 distinct genes that regulate specific normal functions [15],[16]NTRK1 encodes TRKA $\rightarrow$ Pain, thermoregulation - NTRK2 encodes TRKB $\rightarrow$ Movement, memory, mood, appetite, body weight - NTRK3 encodes TRKC $\rightarrow$ Proprioception.Recurrent chromosomal fusion events have been identified across diverse pediatric and adult cancers[16],17].

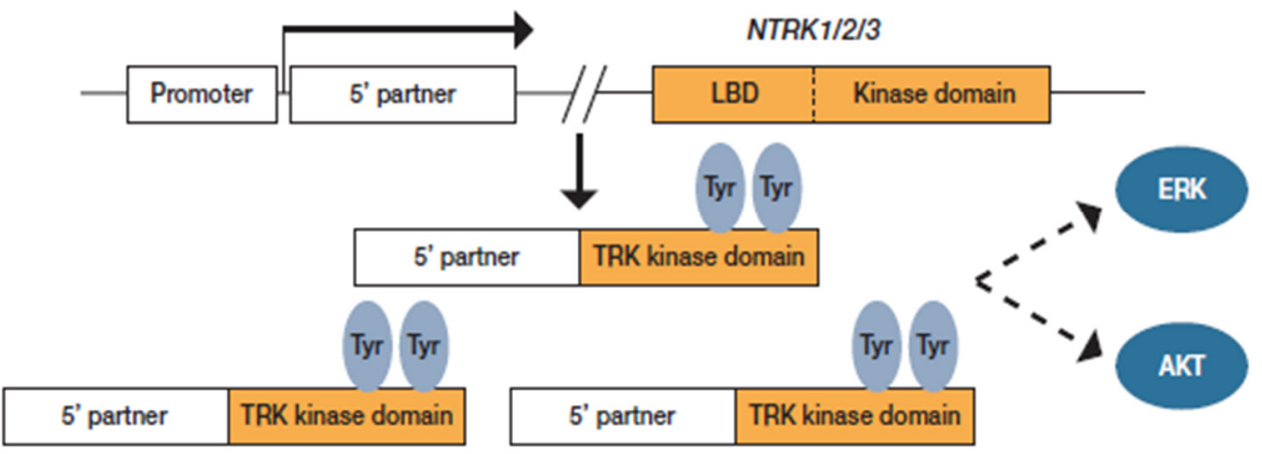

Figure 01: Mechanism of action of the drug. Here,LBD, ligand binding domain 


\subsection{Larotrectinib is efficacious regardless of tumor type (INV):}

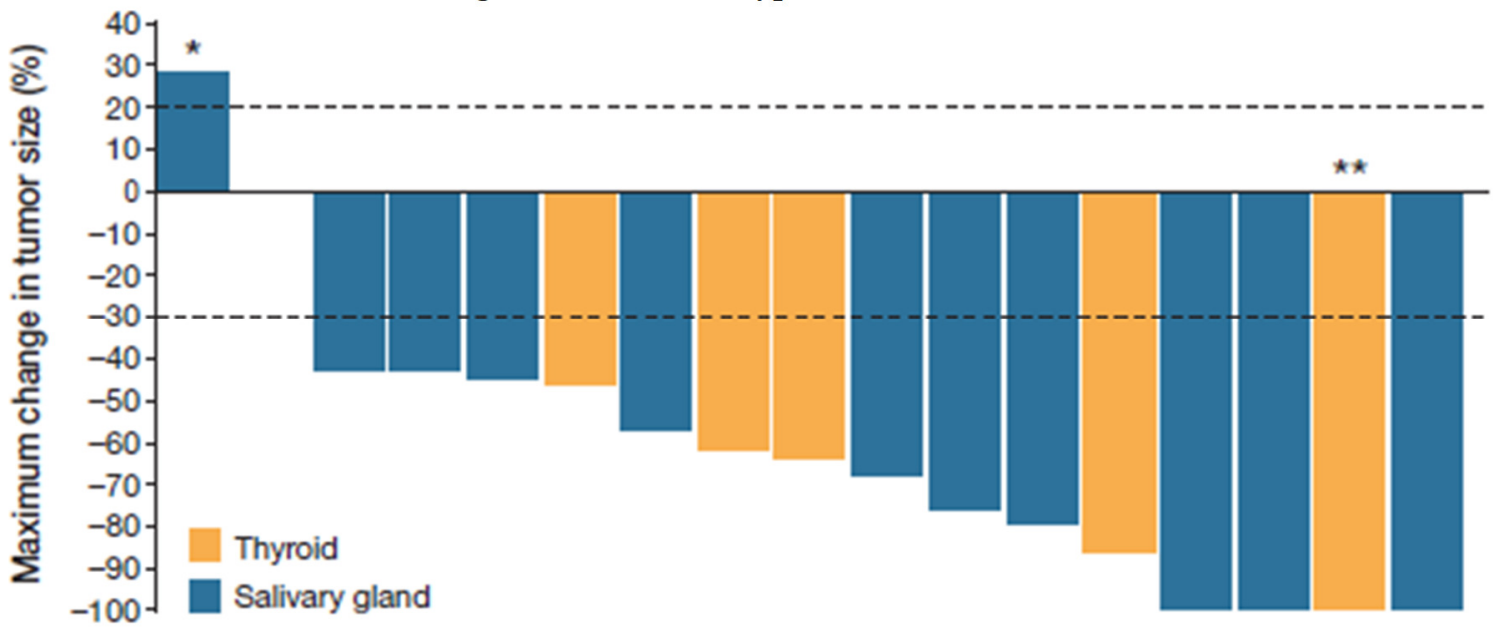

Figure 02: *Patient had a mutation at enrollment known to confer resistance to TRK inhibition due to prior TRK inhibitor therapy **Patient with anaplastic TC who achieved a complete response in target lesions 2 patients with TC (not shown) had non-measurable disease at baseline INV, Investigator response assessment

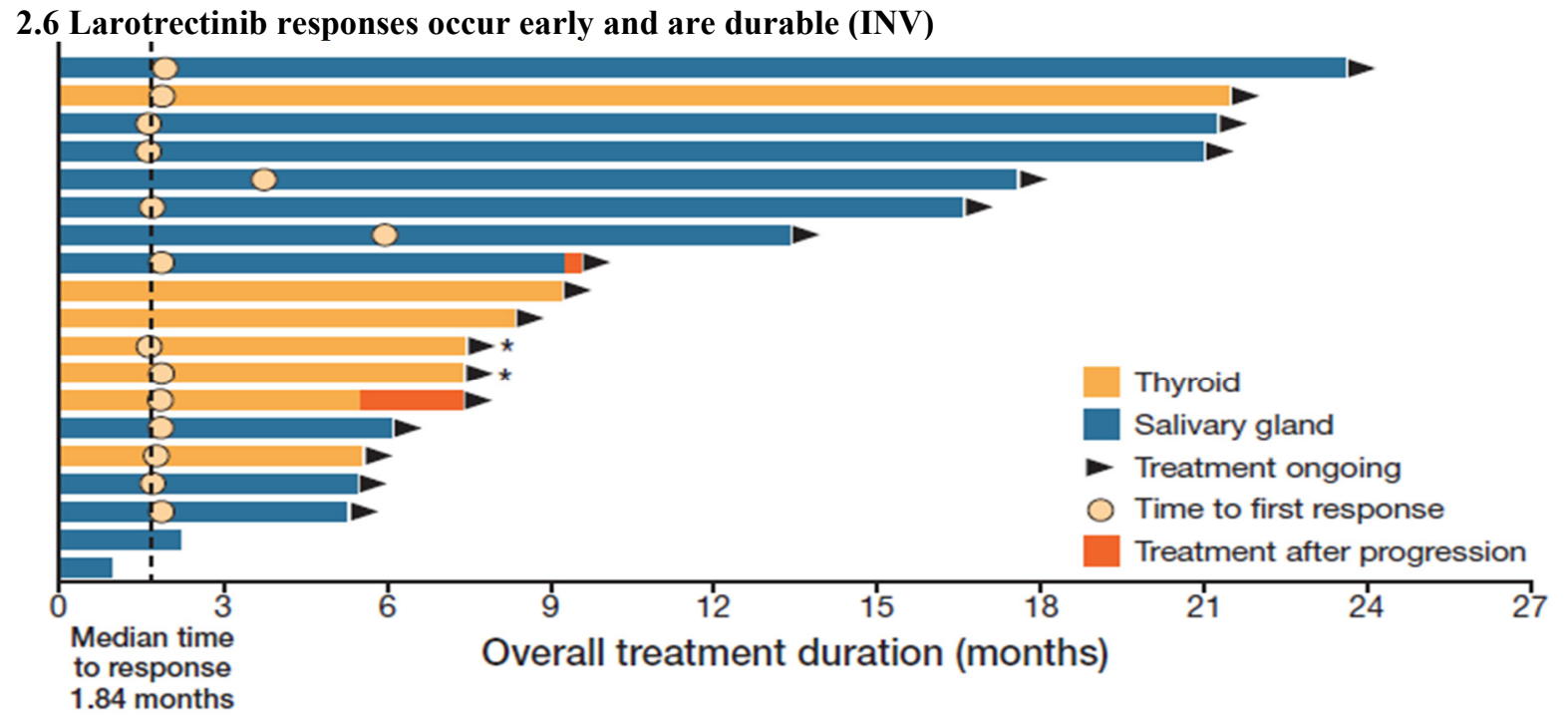

Figure 03: *Patient enrolled with non-measurable disease, assessed as non-CR/non-PD INV, Investigator response assessment.

\section{Experienced, these tend to have a severe expression}

Abnormal Liver Function Tests, Anemia and Disorder of Nerve

If experienced, these tend to have a Less Severe expression

Constipation, Cough, Diarrhea, Dizzy, Feel Like Throwing Up, Low Energy and Throwing Up.

If experienced, these tend to have a severe expression (Infrequent side effects)

Cellulitis, Falling, High Blood Pressure, Low Amount Of Sodium In The Blood, Sepsis Caused By Bacteria, Yellowing Of Skin Or Eyes From Liver Problems

If experienced, these tend to have a Less Severe expression

Abnormal Manner Of Walking, Backache, Delirium, Dysarthria, Extreme Loss Of Body Water, Feeling Faint, Feeling Weak, Fever, High Blood Levels Of The Amylase Enzyme, High Blood Levels Of The Lipase Enzyme, Intense Abdominal Pain, Joint Pain, Loss Of Appetite, Loss Of Memory, Muscle Pain, Muscle Weakness, Numbness And Tingling, Pain, Shaking Extremities, Stuffy Nose, Trouble Breathing and Weight Gain.

If experienced, these tend to have a severe expression (Rare side effects)

Acute Myeloid Leukemia, Enterocutaneous Fistula, Escape Of Fluid Into The Lungs, Fluid Accumulation In The Brain, Fluid In The Covering Of The Heart Or Pericardium, Hole In The Intestine, Stomach Or Intestine Blockage. 


\section{Computational Procedure}

\subsection{Materials and Methods}

The gem structures of proteins 5FDX, 5KMO, 4Z32, 1ZBH, 3RTY gathering of homo insightfulness have been gathered from RCSB PDB protein databank [5]. Every one of the proteins were improved for the experimentation utilizing protein arrangement module of Schrodinger's Maestro demonstrating suit [6]. Hydrogen molecules were included trailed by vitality minimization and streamlining by Chem3DPro12.0 program. The docking was finished by PyRx and chose non-covalent communications among ligands and proteins were gotten by Discovery Studio 2016. MedChem Designer, admetSAR@LMMD, PreADMET was utilized for ADMET examination of the recognized compound and PreADMET was additionally utilized for the poisonous quality investigation of the distinguished compound. WebMO Login. Rendition: 18.0.002e was utilized for HOMO-LUMO examination of the recognized compound.

\subsection{Protein Preparation}

Before docking, heteroatoms, lipids and water molecules were withdrawn from the crystal structure using PyMOl (version 1.7.4.5) [7]. Geometry and energy minimization of the crystal structure were carried out with Swiss-PDB Viewer (version 4.1.0) employing GROMOS96 force field 12. Finally the ligand and protein structures were saved as PDB files. Proteins were selected for Larotrectinib by Swiss Target Predictor.

\subsection{Ligands Optimization}

All predictions were done by using Gaussian view 09 and Chem3D Pro12.0 program packages [8]. Initial threedimensional geometry of chair forms of Larotrectinib was retrieved. The main drug was modified with $\mathrm{F}, \mathrm{Cl}, \mathrm{Br}$, $\mathrm{I}$ and $-\mathrm{CF}_{3}$ functional groups. These structures were fully optimized by density functional theory. Midix basis set was employed for $-\mathrm{Cl},-\mathrm{Br}$ and $-\mathrm{I}$ substituted ligands, while $6-311 \mathrm{G}(\mathrm{d}, \mathrm{p})$ basis set was used for the parent drug and the $-\mathrm{F}$ and $-\mathrm{CF}_{3}$ modified derivatives in Figure 1.

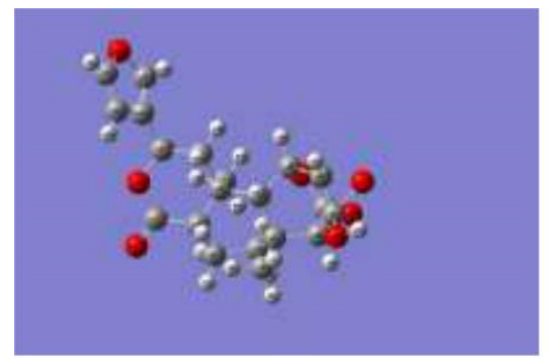

D- Larotrectinib chair

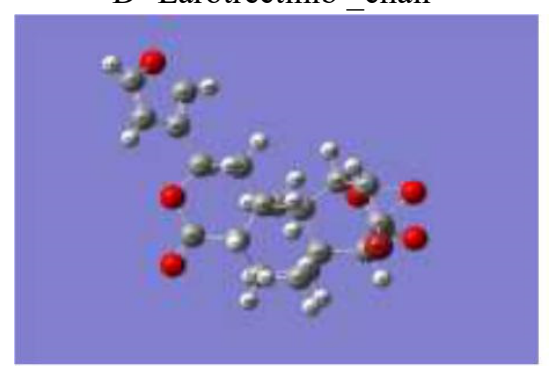

$\mathrm{D}-\mathrm{Br}$ chair

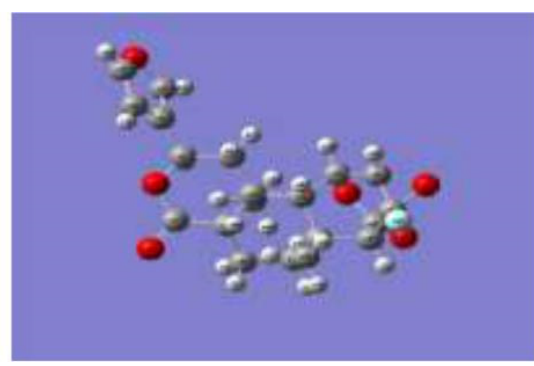

D-F chair

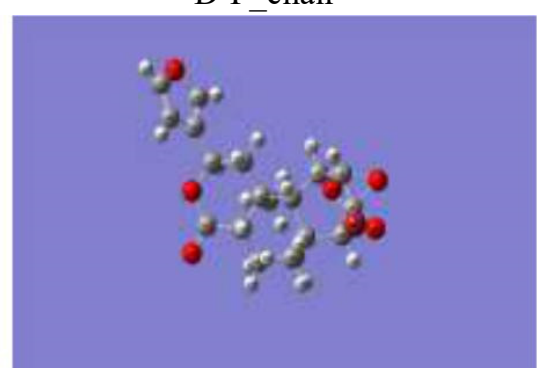

D-I chair

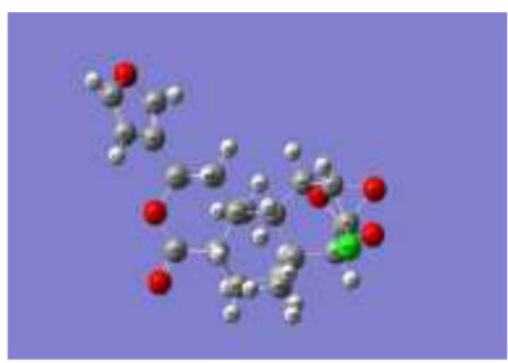

D-Cl chair

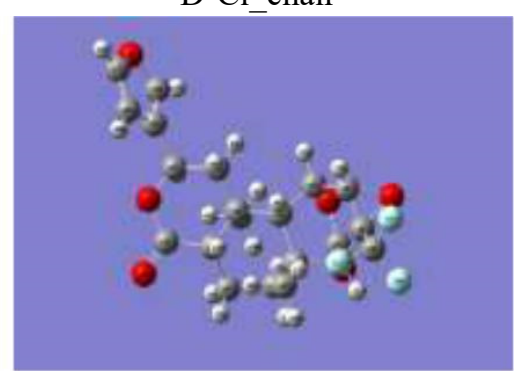

$\mathrm{D}_{-} \mathrm{CF}_{3} \_$chair

Figure 04: Preparation of derivatives. 

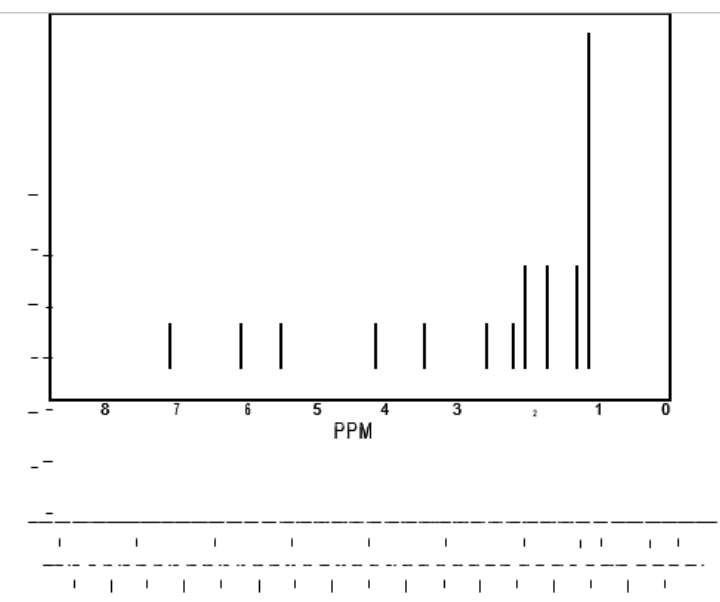

D-Larotrctinib

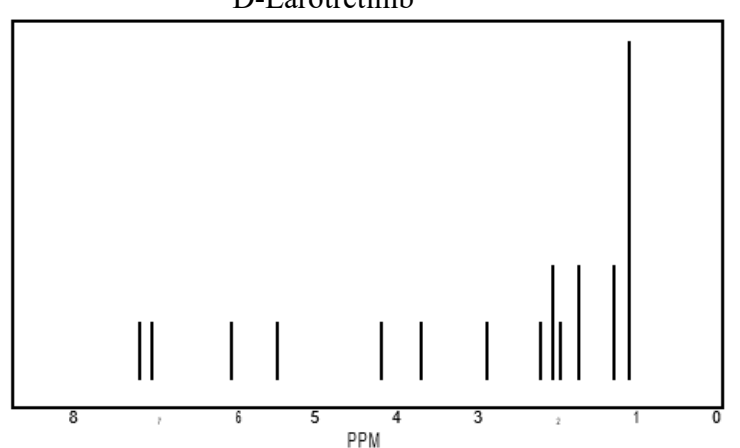

$\mathrm{D}-\mathrm{Cl}$

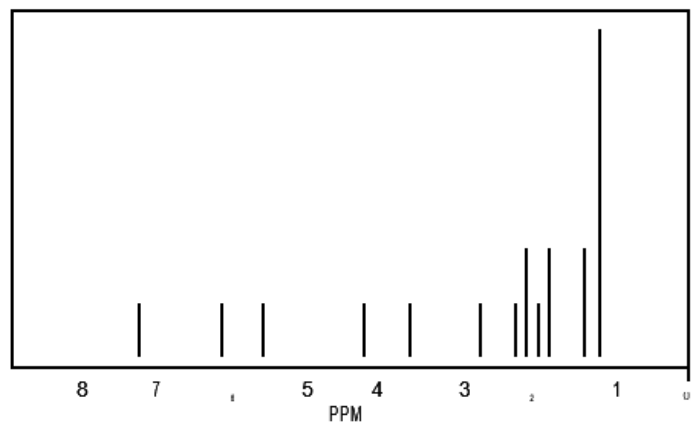

D-I

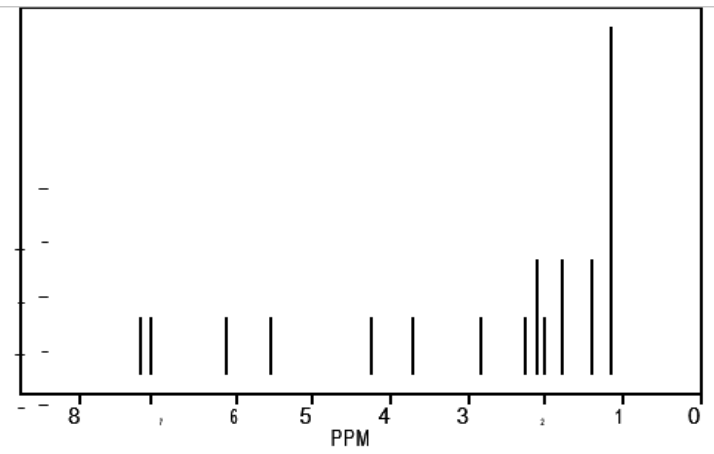

- -
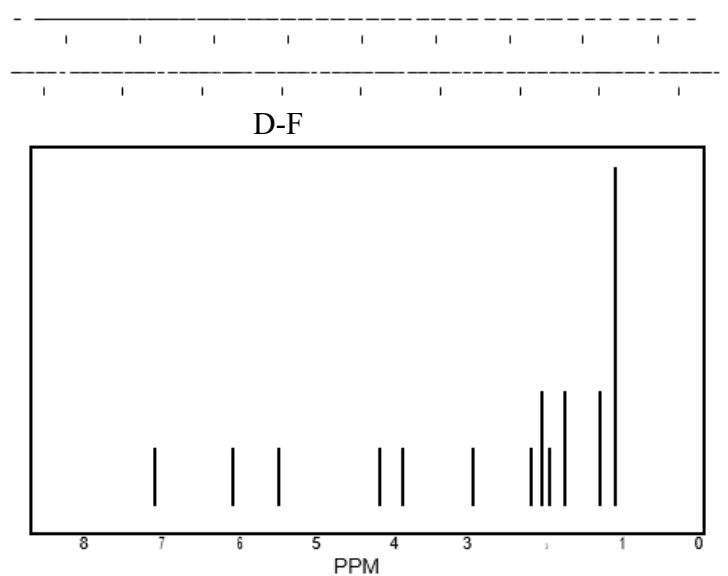

$\mathrm{D}-\mathrm{Br}$

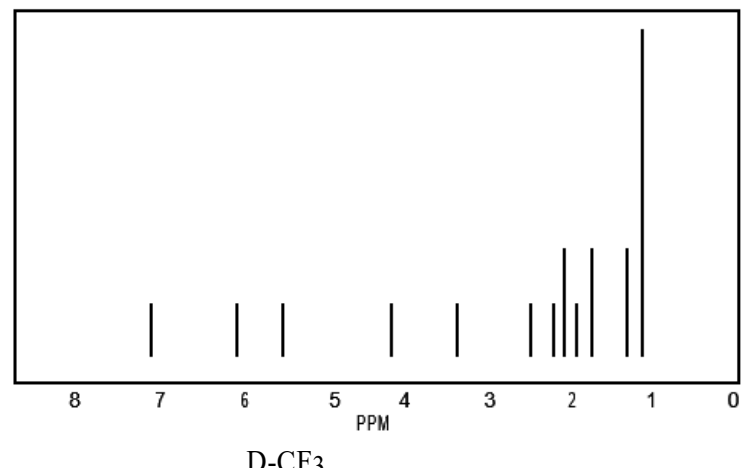

D-CF3

Figure 2. Identification of ligands by Chem NMR H-1 estimation.

\section{Results and Discussion}

\subsection{Admet Analysis of the Identified Compounds}

Ligand screening was based on hierarchal screening approach in docking. High through virtual screening (HTVS) and MedChem Designer, admetSAR@LMMD, PreADMET softwares were used for absorption, distribution, metabolism, and excreation and toxicity analysis of the halogen-modified derivatives. According to MedChem Designer, in generally, lipophilicity is the logarithm value of the partition coefficient $\mathrm{P}(\log \mathrm{P})$ between octanol and water (buffer), which explains the partition of the unionized (neutral) form of the compound, whereas $\log \mathrm{D}$ describes the total partition of both the ionized and the unionized forms of the compound [9]. Compounds have logp value more than 5 indicating their lipophilic proprieties, whereas compounds D-F, D-Cl, D-Br and its analogues D-I and D-CF3 showed low $\log \mathrm{P}$ values less than 5 expressing their hydrophilic nature. MlogP (Moriguchi octanol-water parti

Coefficient) is well known and is traditionally used in QSAR model structure analysis. It describes the lipophilicity of a compound, which indicates the penetration of the compound from aqueous solutions to lipid-rich zones. Moriguchi's $\log \mathrm{P}(\mathrm{MLogP})$ of greater than 4.15 suggests that the compound would be poorly absorbed [10]. The calculated MLogP of compounds D-F, D-Cl, D-Br and its analogues D-I and D-CF3 showed calculated MLogP value significantly less than 4.15, suggesting that these compounds would be easily absorbed. (Table 13). 
Table 1. Medchem designer admet values.

\begin{tabular}{lllllll}
\hline ID & D & D-F & D-Cl & D-Br & D-CF & D-I \\
\hline MlogP & 0.99 & 1.56 & 1.45 & 1.67 & 1.89 & 1.87 \\
S $+\log P$ & 1.27 & 2.46 & 2.05 & 2.57 & 2.71 & 2.86 \\
S $+\log D$ & 1.27 & 2.46 & 2.05 & 2.57 & 2.71 & 2.86 \\
MWt & 374.39 & 392.83 & 376.38 & 437.29 & 426.39 & 484.29 \\
\hline
\end{tabular}

Table 2. AadmetSAR@LMMD-ADMET values.

AdmetSAR@LMMD was used for the prediction of following parameters of the compound.

\begin{tabular}{lcccccc}
\hline Parameters & $\mathrm{D}$ & $\mathrm{D}-\mathrm{F}$ & $\mathrm{D}-\mathrm{Cl}$ & $\mathrm{D}-\mathrm{Br}$ & $\mathrm{D}-\mathrm{I}$ & $\mathrm{D}-\mathrm{CF} 3$ \\
& & & & & & \\
\hline Blood Brain Barrier & 0.888 & 0.938 & 0.930 & 0.927 & 0.914 & 0.941 \\
Human Intestinal & 0.969 & 0.996 & 0.996 & 0.994 & 0.979 & 1.000 \\
$\begin{array}{l}\text { Absorption } \\
\text { Caco-2 Permeability }\end{array}$ & 0.571 & 0.500 & 0.500 & 0.509 & 0.509 & 0.532 \\
$\begin{array}{l}\text { P-glycoprotein Inhibitor } \\
\text { Human Ether a-go-go- }\end{array}$ & 0.723 & 0.500 & 0.550 & 0.692 & 0.570 & 0.857 \\
Related (hERG) Gene & 0.991 & 0.991 & 0.984 & 0.990 & 0.987 & 0.983 \\
Inhibition & & & & & & \\
Acute Oral Toxicity & 0.349 & 0.494 & 0.463 & 0.4978 & 0.497 & 0.404 \\
Rat Acute Toxicity, & 3.239 & 2.692 & 2.651 & 2.682 & 2.649 & 2.920 \\
LD50 (mol/kg) & & & & & & -5.001 \\
Aqueous Solubility & -4.097 & -4.354 & -4.545 & -4.470 & -4.511 & -5.000 \\
\hline
\end{tabular}

Table 3. PreADMET-ADMET Values.

PreADMET was used for the ADMET analysis of the following identified compound.

\begin{tabular}{lllllll}
\hline Parameters & \multicolumn{1}{c}{$\mathrm{D}$} & $\mathrm{D}-\mathrm{F}$ & $\mathrm{D}-\mathrm{Cl}$ & $\mathrm{D}-\mathrm{Br}$ & $\mathrm{D}-\mathrm{I}$ & $\mathrm{D}-\mathrm{CF}_{3}$ \\
\hline Buffer_solubility_mg/L & 79580.6 & 79580.6 & 57511.4 & 3330.93 & 926.62 & 7081.01 \\
Plasma_Protein_Binding & 89.91 & 89.91 & 82.43 & 99.61 & 100.00 & 92.08 \\
Pure_water_solubility_mg & 7.78 & 7.78 & 30.00 & 4.80 & 1.61 & 6.44 \\
Skin_Permeability & -4.32 & -4.32 & -4.28 & -4.33 & -4.33 & -3.53 \\
& & & & & & \\
\hline
\end{tabular}

\subsection{Toxicity Analysis of Compounds}

PreADMET was also used for the toxicity analysis of the following identified compound. Toxicity of all the compounds were predicted by PreADMET suggesting that all the compounds having toxicity less than 1.0 given in Table 4.

\subsection{Homo-Lumo, Gap, Hardness and Softness Analysis}

Hardness $(\eta)$ and softness (S) of all drugs were also calculated from the energies of frontier HOMOs (highest occupied molecular orbitals) and LUMOs (lowest unoccupied molecular orbitals). Hardness ( $\eta$ ) and softness (S) of the drugs calculated according to the following equation (Pearson 1986, 1995) [11], [12].

$$
\begin{gathered}
\eta=\frac{\varepsilon L U M O-\varepsilon H O M O}{2} \\
S=\frac{1}{\eta}
\end{gathered}
$$

D- $\mathrm{CF}_{3}$ Larotrectinib showed small gap value 1.891, hardness 0.945 that was very lower and softness value 1.057 that was good for absorption in Table 5. So, $\mathrm{D}-\mathrm{CF}_{3}$ Larotrectinib was the best among the derivatives. 
Table 5. HOMO-LUMO, Gap, Hardness and Softness analysis.

\begin{tabular}{llllll}
\hline Molecules & ${ }^{8}$ HOMO & LUMO & Gap & Hardness & S(Softness) \\
\hline D_Chair & -7.871 & -2.31 & 5.561 & 2.780 & 0.3596 \\
D-F_Chair & -7.718 & -1.226 & 6.492 & 3.246 & 0.308 \\
D-Cl-Chair & -7.919 & -2.305 & 5.614 & 2.807 & 0.3562 \\
D-Br_Chair & -7.839 & -0.746 & 7.093 & 3.546 & 0.5639 \\
D-I_Chair & -6.641 & -1.618 & 5.023 & 2.511 & 0.3981 \\
D-CF3_Chair & -4.222 & -2.331 & 1.891 & 0.945 & 1.0576 \\
\hline
\end{tabular}

4.4. Free energy of binding values $\left(\mathrm{Kcalmol}^{-1}\right)$ for ligand - Larotrectinib (at chair form) systems obtained from docking.

The binding affinity of D- Larotrectinib showed the value -8.9 for TYROSINE KINASE RECEPTOR (3RTY) So, D-CF 3 -3RTY showed the lowest binding energy in Table 7.

Table 6. Free energy of binding values $\left(\mathrm{Kcalmol}^{-1}\right)$ for ligand -Tyrosine kinaase receptor (TRK)

\begin{tabular}{cllllll}
\hline Protein & D (chair) & D-F (chair) & D-Cl (chair) & D-Br (chair) & D-I(chair) & D-CF3 $^{\text {(chair) }}$ \\
\hline 5FDX & -7.9 & -8.4 & -8.4 & -7.9 & -7.8 & -8.5 \\
5KMD & -8.1 & -8.0 & -8.1 & -8.0 & -8.1 & -8.2 \\
44Z32 & -8.2 & -8.2 & -8.2 & -8.2 & -8.1 & -8.5 \\
1ZBH & -8.1 & -8.3 & -8.1 & -8.0 & -7.8 & -8.4 \\
3RTY & -8.8 & -8.9 & -8.7 & -8.3 & -7.8 & -8.9 \\
\hline
\end{tabular}

\subsection{Binding Site and Docking}

The active binding pocket of Larotrectinib were predicted by CASTP. The binding site residues predicted by CASTP for Larotrectinib (D-3RTY, D-F-3RTY, D-Cl-3RTY, D-CF $3-3 R T Y$ ) were used for grid generation. The docked pose of lowest binding free energy conformer with the respective protein was analyzed using PyMOL Molecular Graphics System (version 1.7.4) [13]. Accelrys Discovery Studio 2016 in Figure 3.

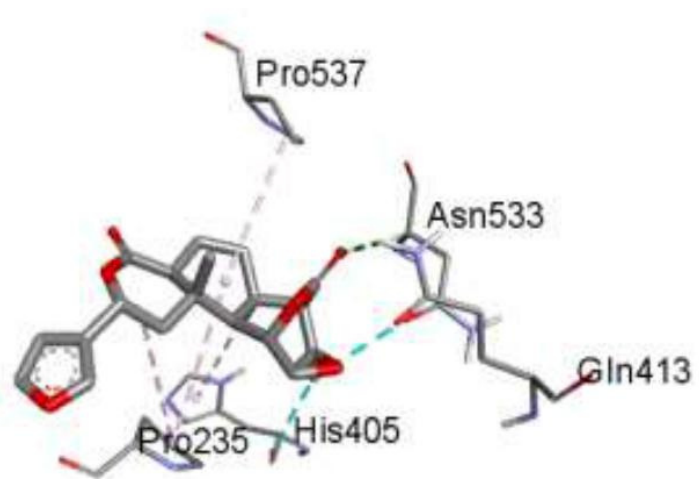

D-3RTY

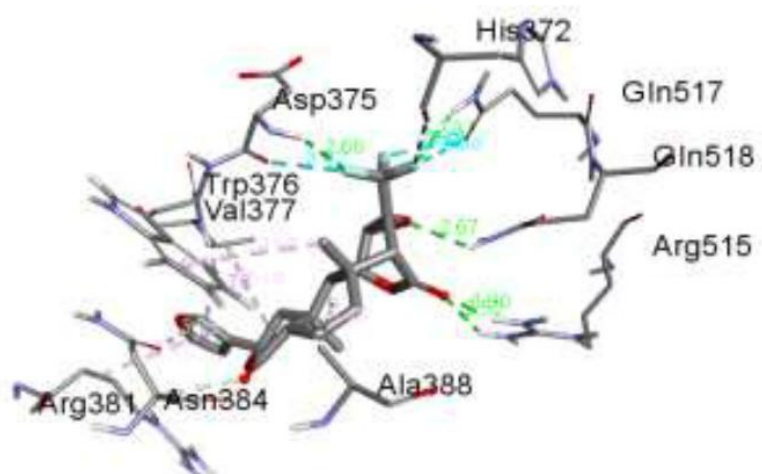

D-CF $3-3 R T Y$

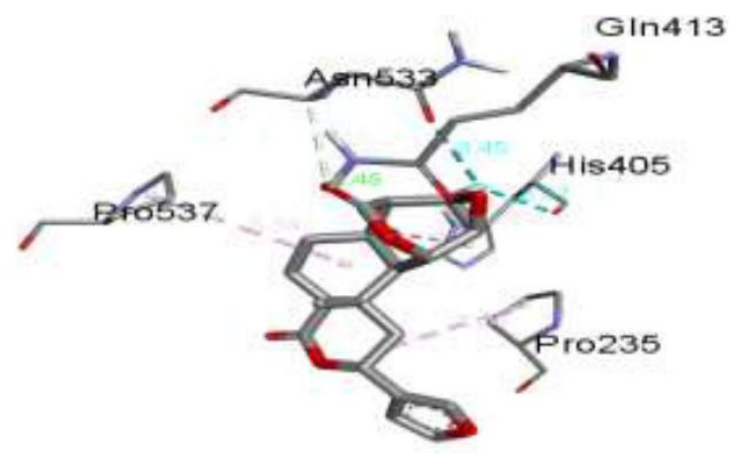

D-F-3RTY

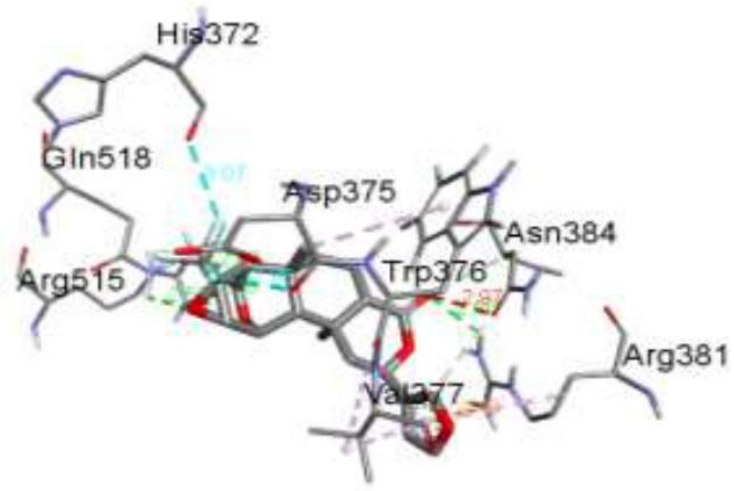

D-CF3 -3RTY

Figure 3. Binding site ligands-proteins. 


\subsection{Selected Non-Covalent Interactions among Chair Ligands D, D-C $f_{3}$ and Larotrectinib (3RTY) Obtained by Discovery Studio}

Selected non-covalent interactions among chair ligands and proteins were given in Table 8-9.

Table 8. Selected non-covalent interactions among chair ligands D, D-F and Larotrectinib (3RTY) obtained via flexible docking.

\begin{tabular}{llllll}
\hline Systems & Contacts & $\begin{array}{l}\text { Bond } \\
\text { distances }(\AA)\end{array}$ & Systems & Contacts & $\begin{array}{l}\text { Bond } \\
\text { distances }(\AA)\end{array}$ \\
\hline D-3RTY & N...H-E (Gln413) & 2.503 & D-F-3RTY & O...H-E (Gln413) & 2.477 \\
& O...C (Asn533) & 3.343 & & O...C (Asn533) & 3.317 \\
& F... (His405) & 3.278 & F...O (Asn533) & 3.446 \\
& Alkyl (Pro235) & 5.076 & Alkyl (Pro235) & 5.037 \\
& Pi...Alkyl (His405) & 4.362 & & Pi...Alkyl (His405) & 4.386 \\
\hline
\end{tabular}

\section{Conclusion:}

TRK combinations can happen in non-papillary TC, and non-MASC, SGC, TRK hindrance with larotrectinib yields high reaction rates, including complete reactions, in teenagers and grown-ups with repetitive pre-treated TRK combination TC and SGC. Responses with larotrectinib treatment are commonly tough [19]. Prolonged larotrectinib treatment is related with insignificant harmfulness and no medication cessations for TEAES. Genomic profiling with tests fit for recognizing TRK combinations ought to be emphatically considered in patients with TC and SGC of all histologies while deciding foundational treatment choices, particularly in the setting of repeat. Our examination affirmed that the coupling fondness of D-CF3 Larotrectinib demonstrated the esteem - 10.1 for tyrosine kinase proteins (3RTY) in correlation with the principle sedate for Larotrectinib.So the tyrosine kinase proteins (3RTY) of larotrectinib are the best proteins. From every one of the information we have examined, asserted that the D, D-CF3 (Larotrectinib) for tyrosine kinase receptor (3RTY) will be the best conformer and will be the best focus as hostile to malignant growth flag transducer protein for the future scientists.

\section{Acknowledgements}

At first, I might want to offer my thanks to Almighty God for giving me the quality and chance to finish my paper inside the timetable time effectively.

I feel glad to express my profound feeling of appreciation to my reverend instructor, guide and chief Dr. Mohammad Torequl Islam and Rezina Rauf, Abul Bashar Ripon Khalipha, Assistant teacher and executive, Department of Pharmacy, Bangabandhu Sheik Mujibur Rahman Science and Technology University, Gopalganj8100, Dhaka, Bangladesh, for her everyday supervision, capable administration, adroit investigation, distinct fascination, hopeful guiding and unremitting reinforcement.

I might want to pass on most profound love and dutifulness to my folks for their help, motivation and managing me all through my life until today, which keeps me solid and firm to do the things I expected to do.

I might likewise want to accept the open door to offer my entire hearted thanks to my kindred scientist companions and precious ones who offered consolation, data, motivation and help amid the time of building the examination report.

We are grateful to Evergreen Research Center (www.esrc-bd.com), Bangladesh and Faculty individuals from Department of Pharmacy of Life Science of Bangabandhu Sheik Mujibur Rahman Science and Technology University to give us support to construct a computational stage.

\section{References}

[1] Hyman et al. J Clin Oncol. 2017; 35 (suppl; abstr LBA2501)

[2] Prasad et al. Cancer. 2016; 122:1097-107.

[3] Greco et al. Mol Cell Endocrinol. 2010; 321:44-9.

[4] Dhama K, Sachan S, Khandia R, Munjal A, Iqbal HMN, Latheef SK, Karthik K, Samad HA, Tiwari R, Dadar M. Recent Pat Endocr Metab Immune Drug Discover. 2017; 10(2):96-111.

[5] RCSB PDB Mobile: iOS and Android mobile apps to provide data access and visualization to the RCSB Protein Data Bank.Quinn GB, Bi C, Christie CH, Pang K, Prlić A, Nakane T, Zardecki C, Voigt M, Berman HM,

[6] Bourne PE, et al.Bioinformatics. 2015 Jan 1; 31(1):126-7. Epub 2014 Sep 2.

[7] Deepa PR, Vandhana S, Muthukumaran S, Umashankar V, Jayanthi U, Krishnakumar S. J Ocul Biol Dis Infor. 2010 Dec; 3(4):117-28. Epub 2011 Nov 24.

[8] Computer-aided drug design platform using PyMOL. Lill MA, Danielson ML.J Comput Aided Mol Des. 2011 Jan; 25(1):13-9. Epub 2010 Oct 30.

[9] Chemistry 380.37 fall 2015 Dr. Jean M. Standard October 5, 2015. 
[10] Cytisine basicity, solvation, $\log P$, and $\log \mathrm{D}$ theoretical determination as tool for bioavailability prediction. Mol Graph Model.

[11] Experimental and computational approaches to estimate solubility and permeability in drug discovery and development settings. Lipinski CA, Lombardo F, Dominy BW, Feeney PJ.Adv Drug Deliv Rev. 2001 Mar 1; 46(1-3):3-26.

[12] Pearson R. G. Absolute electronegativity and hardness correlated with molecular orbital theory. Proceedings of the National Academy of Sciences of the United States of America. 1986; 83(22):84408441.Doi:10.1073/pnas.83.22.8440.

[13] Pearson R. G. The HSAB principle - more quantitative aspects. Inorganica Chimica Acta. 1995; 240(1-2):9398.

[14] Synthesis, antimalarial activity, heme binding and docking studies of N-substituted 4-aminoquinolinepyrimidine molecular hybrids. Eur J Med Chem. 2017 Mar 31; 129:175-185. Doi:10.1016/j.ejmech.2017.02.024. Epub 2017 Feb 15.

[15] Skalova. Head Neck Pathol. 2013; 7:S30-6.

[16] Crowley et al. Cell. 1994; 76:1001-11.

[17] Huang and Reichardt. Annu Rev Neurosci. 2001; 24:677-736.

[18] Ardini et al. Mol Oncol. 2014; 8:1495-507.

[19] Chen et al. Anticancer Res. 2014; 34:1595-600. 